\title{
Dual identities
}

Some people's blood contains cells from a sibling. Others are two individuals rolled into one. Yet more carry a distinct mutation in only parts of their bodies. Helen Pearson investigates chimaerism and mosaicism.

E ght years ago in Britain, a boy was born who, genetically, was two people. He was formed when two eggs, fertilized by two different sperm, fused into one embryo inside his mother's womb.

He was an unremarkable baby. But as a toddler, doctors discovered that he was a hermaphrodite - what was originally diagnosed as an undescended testis turned out to be an ovary, a fallopian tube and part of a uterus. Further investigation revealed that some parts of his body were genetically female but the rest, which contained a different combination of his parents' genes, was male ${ }^{1}$.

The boy, who was otherwise healthy, is one of only a handful of known true human chimaeras - people carrying tissues that originated in two separate embryos. More common are mosaics, who have patches of tissue that differ genetically from the rest of their body, thanks to a mutation or chromosomal anomaly that arose early in embryological development.

The frequencies of chimaerism and mosaicism are unknown, but doctors might benefit from a better understanding of both conditions. In recent years, tantalizing hints have emerged that pockets of genetically mismatched cells may contribute to conditions as common as infertility, autism and Alzheimer's disease. "I think mosaicism has been neglected as an underlying cause of disease," says Huntington Potter, who works on the genetics of Alzheimer's at the University of South Florida in Tampa.

And if chimaeras and mosaics are more common than we realize, they will complicate future efforts to tailor drug treatments to people's individual genetic constitutions. Two genetically different tissues in one body might produce an unpredictable

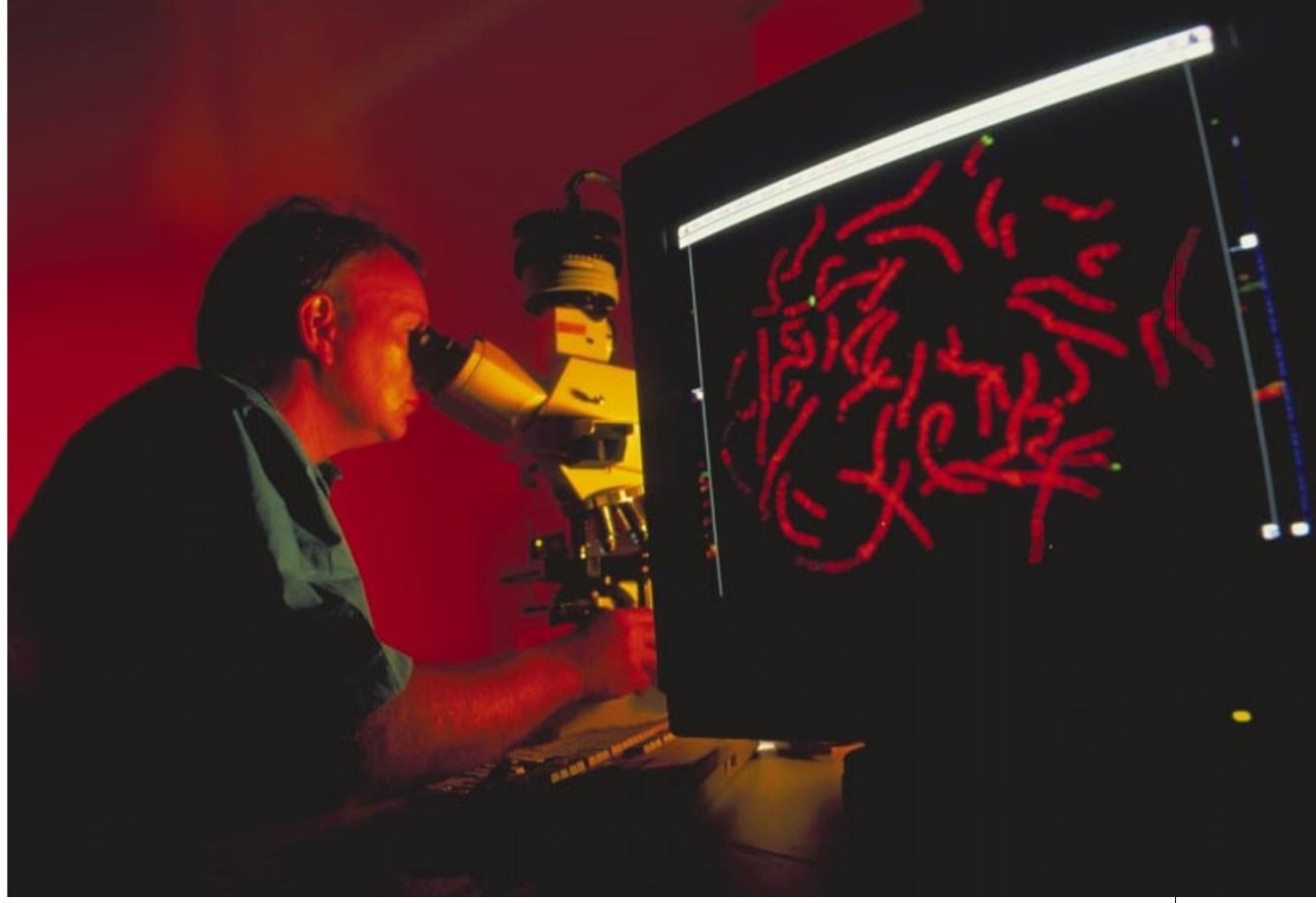

Cryptic conditions: chimaerism and mosaicism are often only spotted by chromosomal analysis.

response to a drug, speculates Roland Wolf, who studies pharmacogenetics at the University of Dundee, UK. "It's completely unknown," he says.

\section{The twin within}

Human chimaerism first came to light with the advent of blood typing - some people, it emerged, have more than one blood group. Most are 'blood chimaeras', nonidentical twins who shared a blood supply in the womb. Those who were not born a twin are thought to be pumping around the remnants of a sibling that died early in gestation and was spontaneously aborted. One British woman, for instance, was

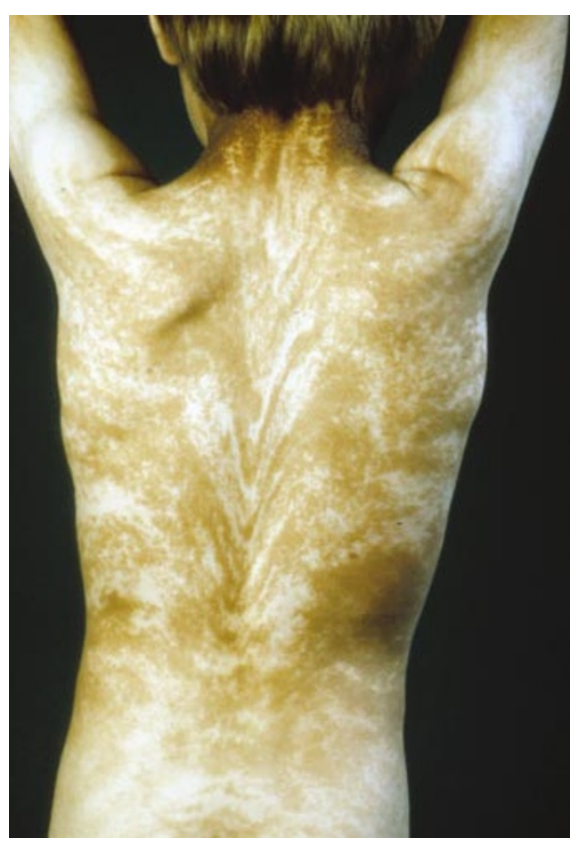

Lines of inquiry: mosaicism causes these unusual pigmented patterns called Blaschko's lines. unaware that she once had a twin until routine blood tests during her pregnancy in the early 1980s revealed a population of chromosomally male blood cells ${ }^{2}$.

Twin embryos often share a blood supply in the placenta, allowing blood stem cells to pass from one embryo and settle in the bone marrow of the other, seeding a lasting source of blood. As a result, as many as $8 \%$ of nonidentical twin pairs have chimaeric blood ${ }^{3}$. And given that most multiple conceptions that result in live births involve the loss of one twin early in pregnancy ${ }^{4}$, there may also be significant numbers of blood chimaeras among single births.

Even more people have 'microchimaerism', carrying smaller numbers of foreign blood cells that may, for instance, have passed between mother and fetus across the placenta, or persist from a blood transfusion. Some researchers argue that the presence of foreign white blood cells might help to explain autoimmune diseases, in which the immune system turns on the body's own tissues.

True chimaeras, in which many tissues are affected, are thought to be very rare, and can form when non-identical twin embryos fuse shortly after fertilization. "If you've got two embryos there's the chance of two becoming one," says clinical geneticist David Bonthron, who led the team at the University of Edinburgh, UK, that reported on the British hermaphrodite boy.

Chimaerism affecting a variety of tissues can also result from other events. In 1995, for instance, Bonthron described another boy who was partially parthenogenetic: cells from his blood and certain other tissues contained none of his father's chromosomes; instead, they featured a duplicated set of one half of his mother's ${ }^{6}$. Although it is not 
unknown for an egg to start developing without being fertilized, fully parthenogenetic human embryos cannot develop to term. Bonthron, now at the University of Leeds, UK, believes that the partially parthenogenetic boy owed his unusual genetic constitution to an egg that spontaneously divided into two cells, one of which was fertilized. The second cell then copied its maternal chromosomes, allowing the resulting chimaera to form a viable embryo.

\section{Hard to spot}

True chimaeras, including both of those identified by Bonthron's team, generally only come to light if they contain male and female cells, when they can cause hermaphroditism or a mismatch between a person's sexual organs and their chromosomal sex as revealed by a blood test. So might the condition be more common than we realize? "I'm convinced that on the streets of London and Hamburg there are many undetected chimaeras," says Rudolf Happle, a dermatologist at the University of Marburg, Germany, who has long been fascinated by mosaicism and chimaerism.

The rise of in vitro fertilization (IVF) is almost certainly bringing more chimaeras into the world. To improve success rates, two or more embryos are placed in the uterus, which explains why women undergoing IVF have up to $25 \%$ more twin pregnancies than usual. More twins means more chimaeras, says Bonthron, who notes that the

\section{think mosaicism has} been neglected as an underlying cause of disease.

Huntington Potter

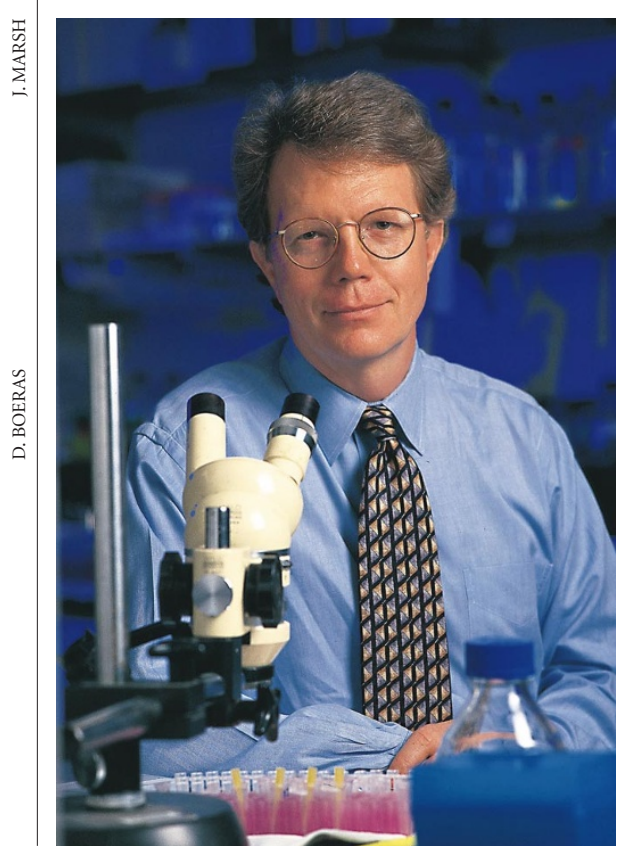

British hermaphrodite boy was an IVF baby ${ }^{1}$.

Mosaicism is more common than chimaerism and is also better studied. Human mosaics arise when a mistake during cell division in the early embryo stops the correct number of chromosomes segregating to each cell, or creates a mutation in a single gene. If this happens in one of the first few cell divisions after fertilization, a large proportion of cells will inherit the defect.

Patchy diseases, in which only regions of tissue are affected, might be caused by mosaicism. Another telltale sign of the condition is a characteristic variation in skin pigmentation that causes patterns called Blaschko's lines, including V-shaped streaks on the back that are sometimes only visible under ultraviolet light.

But pinning down the specific mutation in a mosaic disease can prove tricky. Leslie Biesecker of the National Human Genome Research Institute in Bethesda, Maryland, is attempting to identify the gene that is mutated in Proteus syndrome. This is the disfiguring condition that the Victorian era 'Elephant Man', Joseph Merrick, is thought to have suffered from. The syndrome's symptoms of patchy tissue overgrowth have led researchers to speculate that it is caused by a mosaic mutation.

\section{Threads and patches}

Biesecker hopes to compare the genes that are active in diseased patches of tissue with those in unaffected areas using DNA microarrays. But obtaining tissue samples from the 100 or so sufferers of Proteus syndrome worldwide is difficult, and the genetic differences between tissues may be slight.

Other researchers suspect that mosaicism could be involved in more common diseases. Wendy Robinson of the University of British Columbia in Vancouver, for instance, is intrigued by the observation that the placenta becomes mosaic in about $2 \%$ of pregnancies. Often, these mosaic placentas contain patches of cells that have an extra chromosome a condition called trisomy. Because both fetus and placenta develop from the same cells, Robinson wonders if many fetuses contain undetected patches of trisomic tissues that persist into adult life. "People could have little pockets of trisomic cells sitting around inside

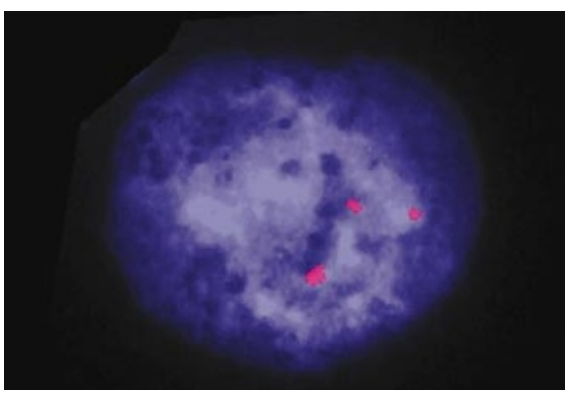

Some Alzheimer's patients have three copies of chromosome 21 (red dots) in certain cells.

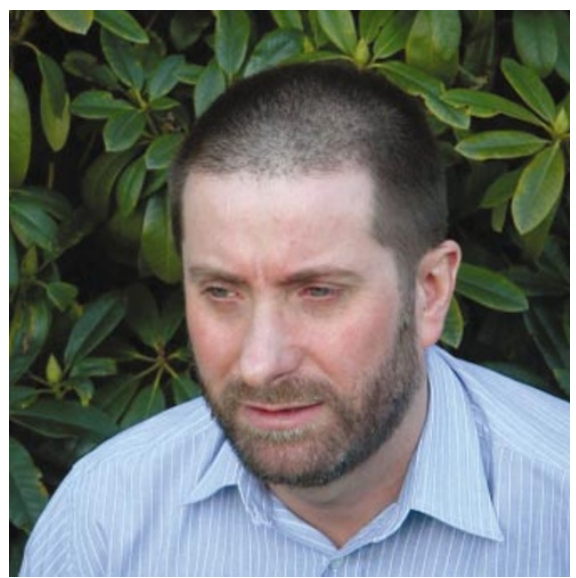

David Bonthron suspects that fertility treatments are increasing the number of human chimaeras.

them that later lead to disease," she suggests. Intriguingly, her team has found that some women who experience recurrent miscarriage carry trisomic cells ${ }^{7}$.

Other researchers theorize that a hidden patch of brain cells with an extra copy of chromosome 21 could be what predisposes some people to Alzheimer's disease. This idea arose from the long-standing observation that people with Down's syndrome, who carry an extra chromosome 21 in all of their cells, develop symptoms of Alzheimer's at an early age. Recently, two research groups, including the South Florida team led by Potter, have found that many patients with Alzheimer's also have low levels of cells with an extra copy of chromosome 21 cells circulating in their blood ${ }^{8,9}$.

Autism is also is being explored for links with mosaicism. In unpublished research, Susan Folstein of Tufts University School of Medicine in Boston examined autistic children under ultraviolet light and found that as many as $10 \%$ of them had pronounced Blaschko's lines. She suspects that a mosaic patch of brain cells, unable to migrate or communicate with its neighbours, might be what causes some cases of autism. But until an underlying mutation can be found, the idea remains unproven.

Given the intriguing results that are starting to emerge, researchers who have studied chimaerism and mosaicism are keen to spread the word so that more attention is paid to the clinical significance of the conditions. At present, they say, most doctors and clinical geneticists are simply not looking. Helen Pearson works in Nature's news syndication team. 1. Strain, L., Dean, J. C. S., Hamilton, M. P. R. \& Bonthron, D. T.

N. Engl. J. Med. 338, 166-169 (1998).

2. Bird, G. W. J. Immunogenet. 9, 317-322 (1982).

3. van Dijk, B. A., Boomsma, D. I. \& de Man, A. J. M.

Am. J. Med. Genet. 61, 264-268 (1996).

4. Boklage, C. E. Int. J. Fertil. 35, 75-94 (1990).

5. Nelson, J. L. Trends Mol. Med. 8, 109-113 (2002).

6. Strain, L., Warner, J. P., Johnston, T. \& Bonthron, D. T. Nature Genet. 11, 164-169 (1995).

Sangha, K. K., Stephenson, M. D., Brown, C. J. \& Robinson, W. P. Am. J. Hum. Genet. 65, 913-917 (1999).

8. Migliore, L. et al. Cytogenet. Cell Genet. 87, 41-46 (1999). 9. Geller, L. N. \& Potter, H. Neurobiol. Dis. 6, 167-179 (1999) 